$$
\text { DOE/ER/45/99--TZ }
$$

\title{
GAMMA SCATTERING IN CONDENSED MATTER WITH HIGH INTENSITY MOSSBAUER. RADIATION
}

DOE/ER/45199--T3

\section{Progress Report}

DE91 001802

\section{A. General review of progress during last 3 year period.}

During the last grant period the successes of the research program can be summarized as follows:

1. Bruce Bullard has completed his thesis research and will receive his doctoral degree from Purdue University in December. A copy of his Ph.D. thesis is enclosed with this renewal proposal. Within the last year five publications related to his doctoral studies have been completed and two of these (submitted to Physical Review) are enclosed as preprints and are included as Appendices A and B of this renewal proposal. Also, Ammar Djedid completed his doctorate at Purdue in May of 1988.

2. Currently three graduate students are carrying out work related to this grant: Ralph Wagoner (Purdue), Scott Dickson (Purdue), and John Day (Purdue). Only one of these is currently being supported (Wagoner) and we seek support for all three, since the success of the proposed research depends on having adequate top-notch students. Some University of Missouri students have participated on the project, but we are presently seeking a well qualified U.M. student to pursue Ph.D. research in this area.

3. The last grant period has resulted in fourteen publications ${ }^{1-14}$. Two of these ${ }^{13,14}$ have just been submitted and the manuscripts are reproduced as Appendices A and B.

4. This work has attracted wide spread attention from the scientific community, and several invited talks in Europe, New Zeland, Australia, and the United States have been given (See listing of invited talks at the end of this section).

5. European scientists have been particularly enthusiastic about this work and one of us (J.G.M.) has received recent letters from Prof. Dr. F. Wagner (Müchen, FRG) and Prof. K. Rubenbauer, Cracow, Poland, proposing collaborative research. Rubenbauer 
is seeking a Fulbright fellowship to spend a year at Purdue working on this D.O.E. sponsored project.

6. Several European scientists have noted that the MURR facility is especially well suited to this work, and that there is not a facility of equal capability for this type of work in Europe. For example, the high flux reactor at Grenoble, using heavy-water, is only suitable for neutron beam experiments in scattering and cannot be used for fabricating high intensity ME sources of the type made at MURR.

\section{List of Publications covering D.O.E. Grant Period 1988-1990}

1. J.G. Mullen, A. Djedid, D. Cowan, G. Schupp, M.L. Crow, Y. Cao, and W.B. Yelon, Physics Letters A127, 242 (1988). "Representation of Lineshape Parameters and Deconvolution of Mössbauer Sepctra."

2. J.G. Mullen, A. Djedid, B. Bullard, G. Schupp, D. Cowan, Y. Cao, M.L. Crow, and W. Yelon, Hyperfine Interactions 40, 123 (1988). "Precise Determination of Mössbauer Lineshape Parameters Including Interference."

3. J.G. Mullen, A. Djedid, G. Schupp, D. Cowan, Y. Cao, M.L. Crow, and W.B. Yelon, Physical Review B37, 3226 (1988). "Fourier-Transform Method for Accurate Analysis of Mössbauer Spectra."

4. G. Haley, J.G. Mullen, and J.M. Honig, Solid State Communications, 69, 285 (1989). "First Order Change in Hyperfine Interaction at the Verwey Transition in Magnetite."

5. M.L. Crow, G. Schupp, W.B. Yelon, J.G. Mullen, and A. Djedid, Physical Review B39, 909 (1989). "Temperature Dependence and Anharmonicity of the Debye-Waller Factor in Sodium Metal Using Mössbauer Gamma-ray Diffraction."

6. B.R. Bullard, J.G. Mullen, and G. Schupp, Hyperfine Interactions 55, 1127 (1990). "Using Lineshape to Precisely Determine / recoil-free Fraction: Application to Tungsten."

7. R. Wagoner, B. Bullard, M. May, S. Dickson, and J.G. Mullen, Hyperfine Interactions 58, 2687 (1990). "Time Dependence of the Source Recoilless Fraction for a Cobalt-57 in Rhodium Source."

8. S.R. Hong, G. Haley, and J.G. Mullen, Hyperfine Interactions 57, 2221 (1990). "Iron Mössbauer Spectra of Lava from Jeju Island and its Similarities to Moon Basalts." 
9. B.R. Bullard and J.G. Mullen, Nucl. Instr. and Meth. B51, 198 (1990).

10. G. Schupp, B. Hammouda, and C.M. Hsueh, Phys. Rev. A41, 5610 (1990).

11. B. Hammouda, G. Schupp, and S. Maglic, Accepted for publication in J. Chem. Phys., "Quasielastic Gamma-Ray Scattering from Polydimethysilocane in Benzene Solutions."

12. J.G. Mullen, B.R. Bullard, and G. Schupp, Proceedings of the Zakopane School of Physics, accepted for publication as a part of the proceedings, World Scientific Publishing.

13. B.R. Bullard, J.G. Mullen, and G. Schupp, Submitted for publication in the Physical Review, "Mössbauer Line-Shape Parameter for ${ }^{183} \mathrm{~W}$ and ${ }^{191} \mathrm{Ir}$ in Metallic Tungsten and Iridium.

14. B.R. Bullard and J.G. Mullen, Submitted for publication in the Physical Review, " Mössbauer Line-Shape Parameters for ${ }^{159} \mathrm{~TB}$ in $\mathrm{TbAl}_{2}$ and $\mathrm{TB}_{4} \mathrm{O}_{7}$.

\section{Ph.D. Theses Resulting From The D.O.E. Sponsored Research}

1. Bruce Bullard, "Mössbauer Studies in Tungsten, iridium, and Terbium Using LineShape Analysis." Purdue University, December 1990.

2. Ammar Djedid, "Precise Lineshape Measurements Using an Analytic Mössbauer Function." Purdue University, May 1988.

3. Lowell Crow, "Mössbauer $\gamma$-ray Scattering in Silicon and Sodium". University of Missouri, December 1987.

\section{Invited Talks J. G. Mullen (1987 - Present)}

1. Fourier Transform Mössbauer Spectroscopy: A New Tool for the study of Fundamental Physics, University of Texas at El Paso, May 26, 1988.

2. Testing Nuclear Dispersion Theory, University of Missouri at Columbia, March 7, 1988.

3. Precision Determination of Mössbauer Lineshape and Quantum Interference, Johns Hopkins University, March 2, 1988.

4. Gamma Ray Scattering, The University of Missouri University Research Reactor Facility, September 29, 1987.

5. True Mössbauer Lineshape and the Determination of Quantum Interference, Purdue University, September 3, 1987. 
6. Precision Determination of Mössbauer Lineshape Parameters Including Quantum Interference, The University of Auckland, New Zealand, August 11, 1987.

7. Lineshape Determination in Mössbauer Spectroscopy, The University of Missouri Research Reactor Facility, June 6, 1987.

8. Mössbauer Diffraction Experiments at the Research Reactor Facility, University of Missouri at Rolla, Chemistry Department, May 2, 1987.

9. Precise Determination of Mössbauer Lineshape Parameters Including Interference, ICAME 87, Melbourne, Australia, August 16-21, 1987.

10. Unique Signatures in Mössbauer Spectroscopy and Deconvolution of Spectra, Jet Propulsion Lab, June 10, 1987.

11. Using the Fourier-Transform Method of ME Spectroscopy for Precision Determination of the Recoilless Fraction, Zakopane School of Physics, May 2, 1990.

12. Measuring Recoilless Fractions and Interference Parameters Using Super-Intense Sources of Tungste:.i-183, Johannes - Gutenberg University, May 8, 1990.

13. Probing Fundamental Physics with the Mössbauer Effect, Purdue University, August 27, 1990.

\section{Invited Talks G. Schupp (1987 - Present)}

1. Dynamics of Polydimethylsiloxane in Benzene Solutions by Quasielastic Gamma-Ray Scattering, San Francisco, November 6, 1989. 


\section{B. Line-Shape Studies}

From the super-intense sources we have been able to fabricate at the MURR facility, we have measured Mössbauer spectra with an accuracy previously unattainable. By fitting our data to an analytic expansion of the convolution integral we have been able to determine the Debye-Waller factor (recoilless fraction) to unprecedented accuracy, accuracies comparable to the best specific heat data and having sufficient precision to complement specific heat data in testing and evaluating lattice dynamics calculations.

When source resonance self absorption (SRSA) is negligible there are six parameters that characterize the $\mathrm{ME}$ line shape. One of these is the inteference parameter, $\beta$. By fitting our high precession data to the analytic expansion of the convolution integral, we have been able to make a quantitative test of the theory ${ }^{1,2}$ of final state effects describing the asymmetric component of the ME spectral line. While our results agree with theory to about 10 to $20 \%$, this difference is very significant in measurements of possible time reversal invariance violation for electromagnetic decay in nuclei, such as the extensive Cal Tech measurements, ${ }^{3}$ where in the case of ${ }^{191}$ Ir the contribution from final state effects is much larger than the sought time reversal phase parameter, $\eta$. For this case the value of $\beta$ (E2) and $\beta$ (M1) are so critical that the two theoretical calculations, which themselves agree to within $5 \%$, that have been carried out lead to different conclusions as to whether or not there is a violation of time reversal symmetry for this case of mixed E2 and M1 decay.

We have carried out these studies of interference and recoilless fraction for ${ }^{183} \mathrm{~W}$ in tungsten metal, ${ }^{191} \mathrm{Ir}$ in iridium metal, and ${ }^{159} \mathrm{~Tb}$ in $\mathrm{TbAl}_{2}$ and $\mathrm{Tb}_{4} \mathrm{O}_{7}$, fitting our data to the convolution integral, and making constrained fits to two more spectra simultaneously to reduce the effects of correlation between the line-shape parameters. ${ }^{4}$

In addition to precise values for interference and recoilless fraction, we find for one of the lineshape parameters, the intrinsic width of the ME transition, values accurate to about $1 \%$, and we believe that our inferred lifetime for the isomer states are the most accurate to date.

These results are described in detail in Appendix A and B, and these two appendices 
have been submitted as manuscripts for consideration as publications in the Physical Review B.

One of the important results to come from our studies of linewhape is a recognition of the importance of source resonance self absorption (SRSA) to the ME spectrum. We have derived an analytic expression ${ }^{5-9}$ for the reduction in the emission recoilless fraction, and we find ${ }^{9}$ that for the commonly used ${ }^{57} \mathrm{Co}$ in rhodium metal sources a surprisingly large time dependerce to the recoilless fraction emitted from such a source, a point which has been ignored by people doing ${ }^{57} \mathrm{Fe}$ Mössbauer spectroscopy.

\section{References}

1. H.C. Goldwire, Jr. and J.P. Hannon, Phys. Rev. B16, 1875 (1977).

2. B.R. Davis, S.E. Koonin, and P. Vogel, Phys. Rev. C22, 1233 (1977).

3. J.L. Gimlett, H.E. Henrikson, H.K. Cheung, and F. Boehm, Phys. Rev. Lett. 42, 354 (1979).

4. B.R. Bullard, J.G. Mullen, and G. Schupp, Hyperfine Interactions 55, 1127 (1990).

5. J.G. Mullen, A. Djedid, G. Schupp, D. Cowan, Y. Cao, M.L. Crow, and W. Yelon, Phys. Rev. B7, 3266 (1988).

6. J.G. Mullen, A. Djedid, B. Bullard, G. Schupp, D. Cowan, Y. Cao, M.L. Crow, and W. Yelon, Hyperfine Interactions 40, 123 (1988).

7. J.G. Mullen, A. Djedid, D. Cowan, G. Schupp, M.L. Crow, Y. Cao, and W.B. Yelon, Phys. Lett. 127, 244 (1988).

8. J. G. Mullen, B. R. Bullard, and G. Schupp, Proceedings of the Zakopane School of Physics, Condensed Matter Studies by Nuclear Method, World Scientific Publishing.

9. R. Wagoner, B. Bullard, M. May, S. Dickson, and J.G. Mullen, Hyperfine Interactions, 58, 2687 (1990). 


\section{Attenuated Source Experiment}

In an attempt to make better measurements of the interference parameter $\beta$, we have used a geometry used earlier by Mössbauer and co-workers. ${ }^{1}$ An absorber of thickness $t_{1}$, is fixed in place relative to the source while an absorber $t_{2}$ is Doppler shifted in the usual manner, so that the gamma beam arriving at the detector has passed through first the stationary and then the moving absorber. The first absorber primarily removes those gamma's centered about the transition energy $E_{o}$, and has less effect on those emitted in the wings, making the transmission signal more sensitive to asymmetries in the regions away from the center of the ME line, and giving a double peak for single line source and absorbers as shown in Figs. 1 and 2 . Since the $\beta$ parameter plays the dominant role in this asymmetry, the method allows a means of focusing on $\beta$.

The actual effect of a non-zero value of $\beta$ can be seen directly in the data shown in Figs 1 and 2. The difference in height of the twin peaks is a result of $\beta \neq 0$. The existence of two peaks rather than the normal single peak is due to the attenution of gammas centered about $E_{o}$. The transmission signal for such an arrangement of absorbers is given by

$$
C(x)=C_{\circ}\left\{1-f_{s o}+\frac{2 f_{o 0}}{\pi} \int d x^{\prime} \frac{e^{-t_{1} \mathcal{L}(2 X)} e^{-t_{2} \mathcal{L}\left(2 x^{\prime}\right)}}{1+4 X^{2}}\right\},
$$

where

$$
X=x^{\prime}-x
$$

and

$$
\mathcal{L}(2 \mathrm{x})=\frac{1-4 \beta \mathrm{x}}{1+4 \mathrm{x}^{2}}
$$

where $x$ is the reduced energy, i.e., in units of the level width $\hbar / \tau$.

To fit this function we have developed a method, described in the next section, that allows direct numerical fitting to any Mössbauer type convolution integral. We are presently testing this method using ${ }^{183} \mathrm{Ta}$ and ${ }^{183} \mathrm{~W}$ metal absorbers. The data for four fixed enriched 
${ }^{183} \mathrm{~W}$ and two moving natural ${ }^{183} \mathrm{~W}$ absorbers is given in Fig. 2. along with the best fit. As in the case of the analytic fit to single lines, good fits can be achieved with a single data set but the correlation between certain parameters leads to large errors in the value of the parameters. The solution to this correlation problem is to fit simultaneously more than one data set at a time. ${ }^{2}$ We cannot only simultaneously fit two or more attenuated data sets, but can also simultaneously fit attenuated sets with data from standard moving and no fixed absorber type geometries. Collection of more ${ }^{183} \mathrm{~W}$ data is presently going on and results in this system are expected within the next three months.

\section{References}

1. R.L. Mössbauer, H.E. Seelbach, B. Persson, M. Bent, G. Longworth, AEC Research and Development Report CALT-63-108.

2. B.R. Bullard, J.G. Mullen, and G. Schupp, Hyperfine Interactions 55, 1127 (1990).

\section{Figure Captions}

Fig 1. Inversion ME spectrum with two enriched stationary absorbers. The solid curve is the best fit to the data based on the convolution integral.

Fig 2. Inversion Me spectrum with four enriched stationary absorbers. The solid curve is the best fit to the data based on the convolution integral. 


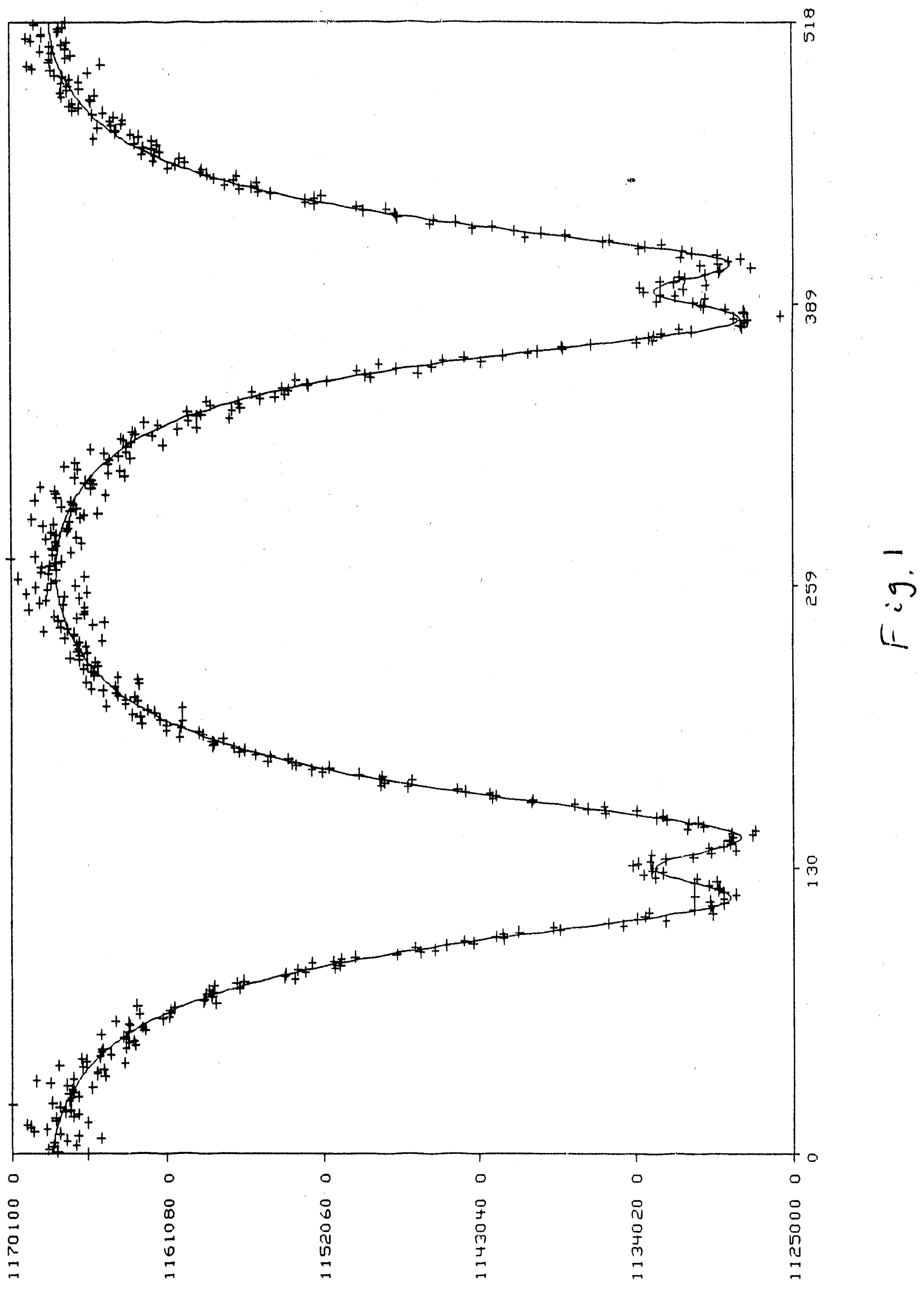




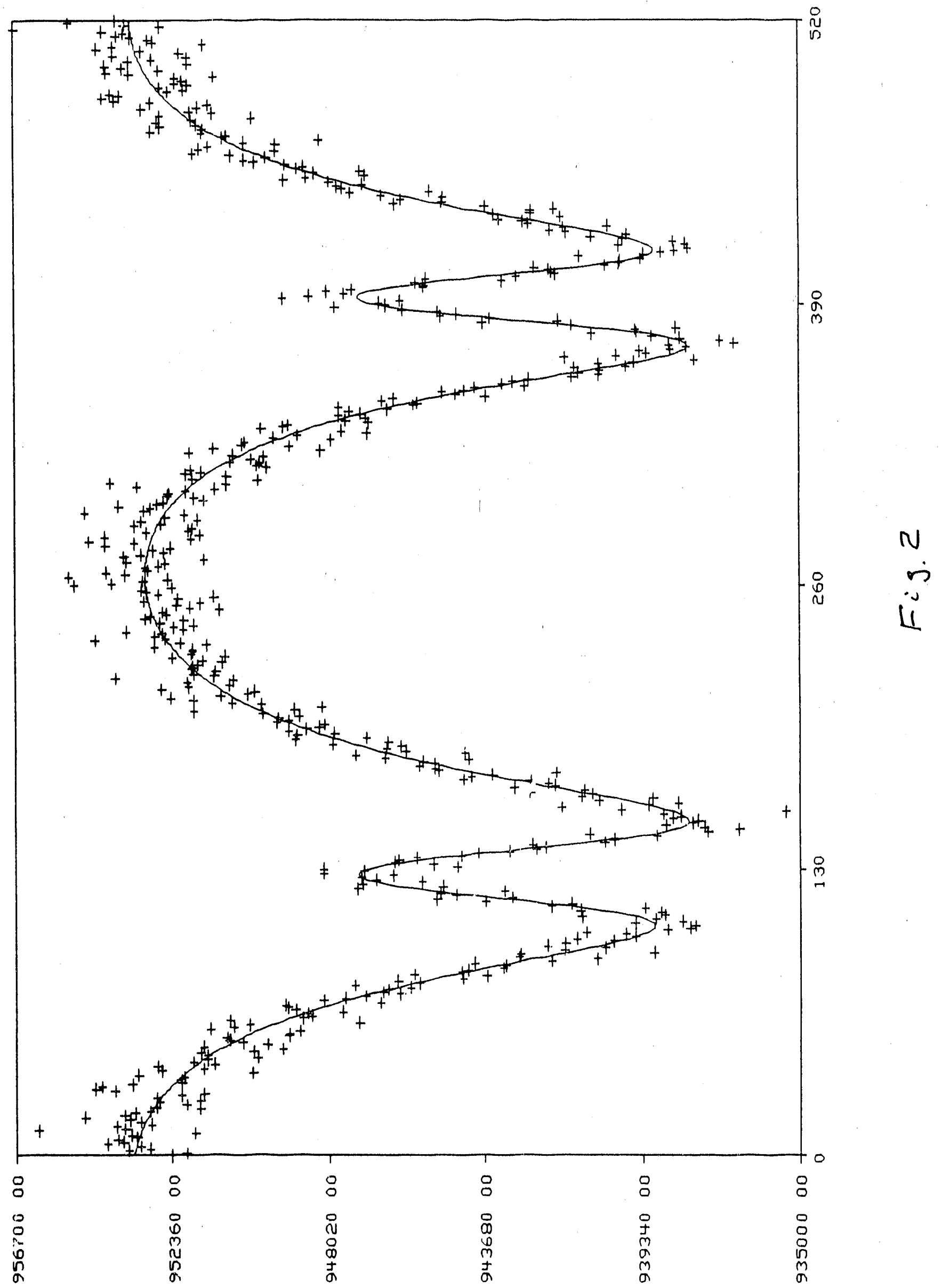




\section{A New Computational Method of Analysis and Its Applications}

We have developed a method of numerical integration which uses a mix of both Weddle's rule and Guassian quadrature using Legendre polynomials, ${ }^{1,2}$ that allows us to accurately fit the data of any Mössbauer experiment. This includes standard cases where source and/or absorber have quadrupole or magnetic splitting as well as $t_{r s}, v_{o}$, and $\beta$; and also unusual cases of varying number, type, or geometry of atsorbers or sources (the attenuated experiment mentioned before being an example).

As long as a mathematical expression can be written down for the transmission signal, and the integrand of the integrals in this expression approach zero as $x$ goes to $\infty$, then our numerical method can be used to fit the data. There is no need to approximate the convolution integral, no need to restrict oneself to thin absorbers or widely shaped split lines, or replacing the absorber or sourcc terms with expansions as a means of replacing the true convolution integral with some simpler version, although an analytic expansion of the convolution integral will reduce the computer time needed to achieve a good fit to data.

We have already tested the method using actual data from the attenuated source experiment, as well as ${ }^{183} \mathrm{~W}$ single line unbroadened data. The fits to the single line case were in excellent agreement with fits made using the analytic function developed earlier. We have also tested it against artificially generated data, including cases with large $t_{r o}$, and cases that included quadrupole splitting in the source, and in all cases accurate results were attained in reasonable computational times. It should be noted that in the case of a single unbroadened line, the analytical method is preferable due to its providing faster convergence. The time consumed, however, using the numerical method is by no means so long as be impractical. For example, in the case of the attenuated source experiment with its complicated convolution integral, data for about 520 points, fit to 7 parameters, took about 4-5 hours of real time

on our ISI/V24, and under 30 minutes on our Stardent Titan P2 mini-supercomputer. This technique is particularly useful in the majority of Mössbauer experiments presently being done which lack any exact analytical expression for their results. 
Two applications of this method which we hope to utilize in the near future, are tests of the interference parameter used in time reversal experiments, and accurate measurement of parameters in various split spectra that are of current interest in Mössbauer studies.

Past time reversal experiments have all relied on theoretical calculations of an interference parameter $\beta_{T}$. This parameter is itself a sum of more basic terms,

$$
\beta_{\mathrm{T}}=\beta(\mathrm{M} 1)-\beta(\mathrm{E} 2)
$$

the same two parameters on which the Mössbauer interference parameter $\beta$ is dependent, i.e.,

$$
\beta=\frac{\beta(\mathrm{M} 1)+\delta^{2} \beta(\mathrm{E} 2)}{1+\delta^{2}},
$$

where $\delta$ is the multiple mixing ratio ${ }^{3}$.

An accurate measurement of $\beta$, though not equivalent to measuring $\beta_{\mathrm{T}}$, would nonethe-less be a consistency check on the theoretical value. In fact, recent experiments ${ }^{4}$ by our group suggest that the theoretical values for $\beta$ in ${ }^{191}$ Ir may be off by as much as $10 \%$. This measurement was made using the standard Mössbauer geometery, and we hope the attenuated geometery will allow an even more accurate measurement of $\beta$, giving a more decisive check on the accuracy of present theoretical models. If the theoretical values are even slightly in error, then all future time reversal work would require either a new theoretical calculation of the value of $\beta_{\mathrm{T}}$.

The great majority of present Mössbauer work involves quadrupole and magnetically split lines. Yet most of these studies still use approximations which have long been recognized as inaccurate. Measurements of $\beta$ have particularly been neglected, and the assumption that $n$ split lines can be fit to $\mathrm{n}$ Lorentzians, is an approximation that ignores the overlap between these lines as well as suffering from the inherent problems of fitting Lorentzians to lines that are not true Lorentzians to begin with. We plan to use our numerical method to draw out all the possible information available in Mössbauer studies, both the nuclear and solid state 
parameters. We believe work in those cases, where the split lines are weakly resolved or not resolved at all, to be of particular importance since it is here that the approximations generally used in the field are most likely to give erroneous results.

\section{References}

1. Erich Gerdau, R. Hollatz, private communication.

2. D. J. Erickson, J. F. Prince, Louis D. Roberts, Phys. Rev. 8C, 1926 (1973).

3. H. C. Goldwire Jr., J. P. Hannon, Phys. Rev 16B, 1875 (1977).

4. B. Bullard, J. G. Sullen, G. Schupp, submitted for publication in the Physical Review B (see Appendix A).

\section{E. Hyperfine Interactions Fitted to the Convolution Integral.}

We have taken measuiements of ${ }^{57} \mathrm{Fe}$ in well characterized magnetite ${ }^{1}$ as well an geological samples taken from Jeju Island. ${ }^{2}$ These samples give interesting hyperfine split ME spectra. Although our first evaluation of these interesting spectra was carried out using the simple Lorentzian function commonly employed in obtaining hyperfine parameters, the residuals indicate that the fits to such data by this commonly used procedure is far from exact and we wish to now employ our direct fits to the convolution integral with a mini super-computer to reevaluate these data correcting for source resonance self absorption (SRSA) and eliminating the saturation effects associated with the use of absorbers of finite thickness.

Two interesting results which have already come from these studies is that the ME spectra for basalts from Jeju Island are remarkably similar to those found in lunar basa!ts. Secondly, we find contrary to all earlier studies that stoichiometric and homogeneous magnetite has a sharp change in the hyperfine field at the Verwey transition characteristic of a first order transition. This result is nicely collaborated by the extensive studies, including specific heat measurements carried out by J. Honig's group.

In addition to attempting to directly fit these hyperfine split spectra to the convolution integral using our new curve fitting procedures, we are also planning the systematic 
deconvolution of these spectra using the Fourier transform technique described in a recent publication. ${ }^{3}$ If this latter technique is successful it will make possible more accurate measurements of hyperfine spectra for all Mössbauer studies of internal magnetic fields and electric field gradients.

\section{References}

1. S.R. Hong, G. Haley, and J.G. Mullen, Hyperfine Interactions 57, 2221 (1990).

2. G. Haley, J.G. Mullen, J.M. Honig, Sol. State Comm 69, 285 (1989).

3. J.G. Mullen, A. Djedid, D. Cowan, G. Schupp, M.L. Crow, Y. Cao, and W.B. Yelon, Physics Letters A127, 242 (1988).

\section{F. Burnout Cross Section of ${ }^{159} \mathrm{Dy}$}

In our attempts to fabricate superintense Mössbauer sources, we have succeeded in several cases. An exception has been the interesting isotope ${ }^{158} \mathrm{Dy}$, whose daughter, ${ }^{158} \mathrm{~Tb}$ has an exceptionally broad width and is well suited to the study of low energy excitations. This surprising result was traced to the large burnout cross section for ${ }^{159} \mathrm{Dy}$ which we have determined $^{1}$ to be $8 \pm 2$ kilobarns. This hugh cross section impedes the production of the Mössbauer parent ${ }^{159} \mathrm{Dy}$ by two orders of magnitude. Using the special facility at Purdue University we have measured the line-shape parameter of this isotope, despite is low intensity. This is possible because this transition is exceptionally clean and experiments can be done directly in the photon beam without the aid of a monochromating crystal filter.

\section{References}

1. B.R. Bullard and J.G. Mullen, Nucl. Inst. Methods B51, 198 (1990). 


\section{G. Anharmonicity in the Temperature Dependence of the Debye-Waller Factor in Sodium Metal.}

The Debye-Waller factor of sodium has been measured ${ }^{1}$ as a function of temperature from 80 to $295 \mathrm{~K}$ using Mössbauer $\gamma$-ray scattering. The high energy resolution provided by this technique allowed experimental separation of the elastic scattering from the inelastic thermal diffuse scattering. The results were compared with the harmonic model using integrations over dispersion curves from the neutron-scattering measurements of Woods et al. ${ }^{2}$ and the lattice-dynamics calculations of Glyde and Taylor. ${ }^{3}$ The Debye-Waller exponent was shown to exceed the harmonic prediction by $23 \%$ at room temperature, and this difference is attributed to anharmonic terms in the interatomic potential.

\section{References}

1. M.L. Crow, G. Schupp, W.B. Yelon, J.G. Mullen, and A. Djedid, Phys. Rev. B39, 909 (1989).

2. A.D.B. Woods, B.N. Brockhouse, R.H. March, A.T. Stewart, and R. Bowers, Phys. Rev. 128, 1112 (1962).

3. H.R. Glyde and R. Taylor, Phys. Rev. B5, 1206 (1972).

\section{H. Recent Scattering Experiments}

The efforts of the last year have been focused in two areas: quasielastic scattering from systems undergoing diffusive neutrons, and scattering close to the transition in a martensitic phase (NiAl). These have provided interesting new results summarized below, and have also made clearer the need for some major improvements in the experimental equipment in order that these studies be performed more reliably and efficiently. These will be explained in the proposal section.

Our Mössbauer diffraction instrument at MURR, with its intense sources, has allowed us to expand into the area of quasielastic gamma-ray scattering (QECS). The $\mu \mathrm{eV}$ energy resolution, as well as the wide range of momentum transfer of this technique, opens new 
possibilities for the study of molecular liquids in general and macromolecular liquids in particular, as well as for solid state diffusion.

Liquids are dominated by thermal Brownian type motion which leads to quasielastic (centered at $\Delta \mathrm{E}=0$ ) scattering processes. Mössbauer studies of diffusion in liquids were first conducted for ${ }^{57} \mathrm{Fe}$ ions dispersed in glycerol by Bunbury et al. ${ }^{1}$ and by Craig and Suttin. ${ }^{2}$ Subsequent to these measurements, Abras and Mullen, ${ }^{3}$ Singh and Mullen ${ }^{4}$ and, most recently, Nienhaus etal., ${ }^{5}$ have also studied different aspects of the scattering by glycerol. Until our work with QEGS, however, these studies have been limited to liquids which included the Mössbauer nuclide. In these studies, the resonant nuclei are moving with the diffusing medium and absorb the incoming radiation according to the velocity of the nuclei. In the present studies, the incoming radiation is Raleigh scattered by the diffusing medium itself and the data interpretation should be unambiguous. Figure 1 summarizes the main tech. niques used to investigate the dynamics of liquids $s^{6,7,8}$ at various characteristic length scales going from the collision dominated hydrodynamic region (very small $Q$ and $\omega$ ) to the free streaming ideal gas limit (very large $Q$ and $\omega$ ). Here, $Q$ and $\omega$ represent the momentum and energy transferred during scattering. Typical characteristic lengths (Angstroms) and time scales (picoseconds) for liquids fall within the $(\mathrm{Q}, \omega)$ windows for neutron scattering, gamma-ray scattering and computer simulation (molecular and Brownian dynamics).

We have conducted three studies on viscous liquids using this new technique. The first was on pentadecane ${ }^{9}$ (published in the Physical Review A, May 1990) and the second was on polydimethylsiloxane (accepted in July 1990 for publication in the Journal of Chemical Physics). In both of these studies, the quasielastic broadening of the Mössbauer velocity spectrum was observed by coherent scattering at the first liquid structure peak. For pentadecane, this broadening was observed versus temperature of the sample in the $12^{\circ} \mathrm{C}$ to $74^{\circ} \mathrm{C}$ range and in the polydimethylsiloxane case, it was observed versus dilution in benzene at room temperature. Also for pentadecane, the width of the quasielastic scattering was measured versus the scattering vector $Q$ (by setting at different positions across the liquid 
structure peak) at room temperature. This latter result showed deGennes narrowing ${ }^{10}$ of the width when setting at the ${ }_{i}$ corresponding to the center of the liquid structure peak.

In the polydimethylsiloxare investigation, part of the scattering was found to be "elastic" (i.e., within the resolution of QEGS) and part quasielastically broadened. Upon dilution in benzene, the "elastic" fraction of the scattering decreased quickly, demonstrating a softening of the stiff degrees of freedom along the backbone of the polymer chain while the scattering from the softer modes broadened further.

The most recent quasielastic scattering measurements by us have been on glycerol in the temperature range from $24^{\circ} \mathrm{C}$ to $113^{\circ} \mathrm{C}$. It is presently in the interpretation and manuscript preparation stage. Based on the earlier work noted on glycerol and the fact that it is a smaller, nearly spherical molecule with a small electric dipole moment, we expected that our results would be straightforward to evaluate. We found, however, a much greater broadening with temperature than was expected (see Fig. 2) and a more complete model is being sought.

The martensitic transition in $\mathrm{NiAl}$ is seen as representative of a large class of materials such $\mathrm{SrTiO}_{3}, \mathrm{KMnF}_{3}$ in which a soft phonon is observed above $\mathrm{T}_{c}$. Below $\mathrm{T}_{c}$ a distorted structure is observed with the new Bragg peaks associated with the soft phonon position. In these systems, a "central peak" is seen at $\mathrm{E}=\mathrm{O}$ at the phonon wavevector and there has been much discussion and investigation of the nature of this central peak. QFGS is one of the best techniques for such an investigation since it has simultaneously excellent energy resolution and access to a large region of reciprocal space.

In $\mathrm{NiAl}$, a soft phonon and central peak is seen at a $\overrightarrow{\mathrm{q}} \simeq \frac{1}{6}-\frac{1}{60}$ (and equivalent positions although the transformed structure appears to correspond to a $\mathrm{q}$ vector closer to $\frac{1}{7}$. Our studies show a more complicated situation in which, rather than satellites along the line between the 220 and 310 with spacing $\sim \frac{1}{7}-\frac{1}{70}$, there are two lines of spots parallel to 
this line, one closer to the origin, the other further away than the $220-310$ line. This structural problem warrants further detailed study.

QEGS measurements were made at the strongest of these satellites as a function of temperature. Up to $\sim 30 \mathrm{~K}$ above $\mathrm{T}_{c}$, the scattering was fully elastic. Above this point, the intensity was too low to follow further. This observation is consistent with other studies which fail to observe broadening or inelasticity in the centrai peak and suggests that the central peak may correspond to static domains, perhaps induced by strains well above $\mathrm{T}_{c}$.

Both the liquid scattering studies and these emphasize the potential of the QEGS technique but both were hampered by limited intensity and less than optimum sources (especially in source cooling). We outline a number of improvements in the next section which will improve these studies substantially.

\section{References}

1. D.St. Bunbury, J.A. Elliot, H.E. Hall, and J.M. Williams, Phys. Lett. 6, 34 (1963).

2. P.P. Craig and N. Suttin, Phys. Rev. Lett. 11, 460 (1963).

3. A. Abros and J.G. Mullen, Phys. Rev. A6, 2343 (1972).

4. K.P. Singh and J.G. Mullen, Phys Rev. A6, 2354 (1972).

5. G.U. Nienhaus, H. Frauenfelder and F. Parak, preprint (1990).

6. J.S. Boon and S. Yip, Molecular Hydrodynamics (McGraw-Hill, New York, 1980).

7. J.P. Hansen and I.R. McDonald, Theory of Simple Liquids (Academic Press, New York, 1976).

8. J.R.D. Copley and S.W. Lovesey, Rep. Prog. Phys. 38, 461 (1975).

9. G. Schupp, B. Hammouda and C.M. Hsueh, Phys. Rev. A41, 5610 (1990).

10. P.G. deGennes, Physica 25, 825 (1959). 


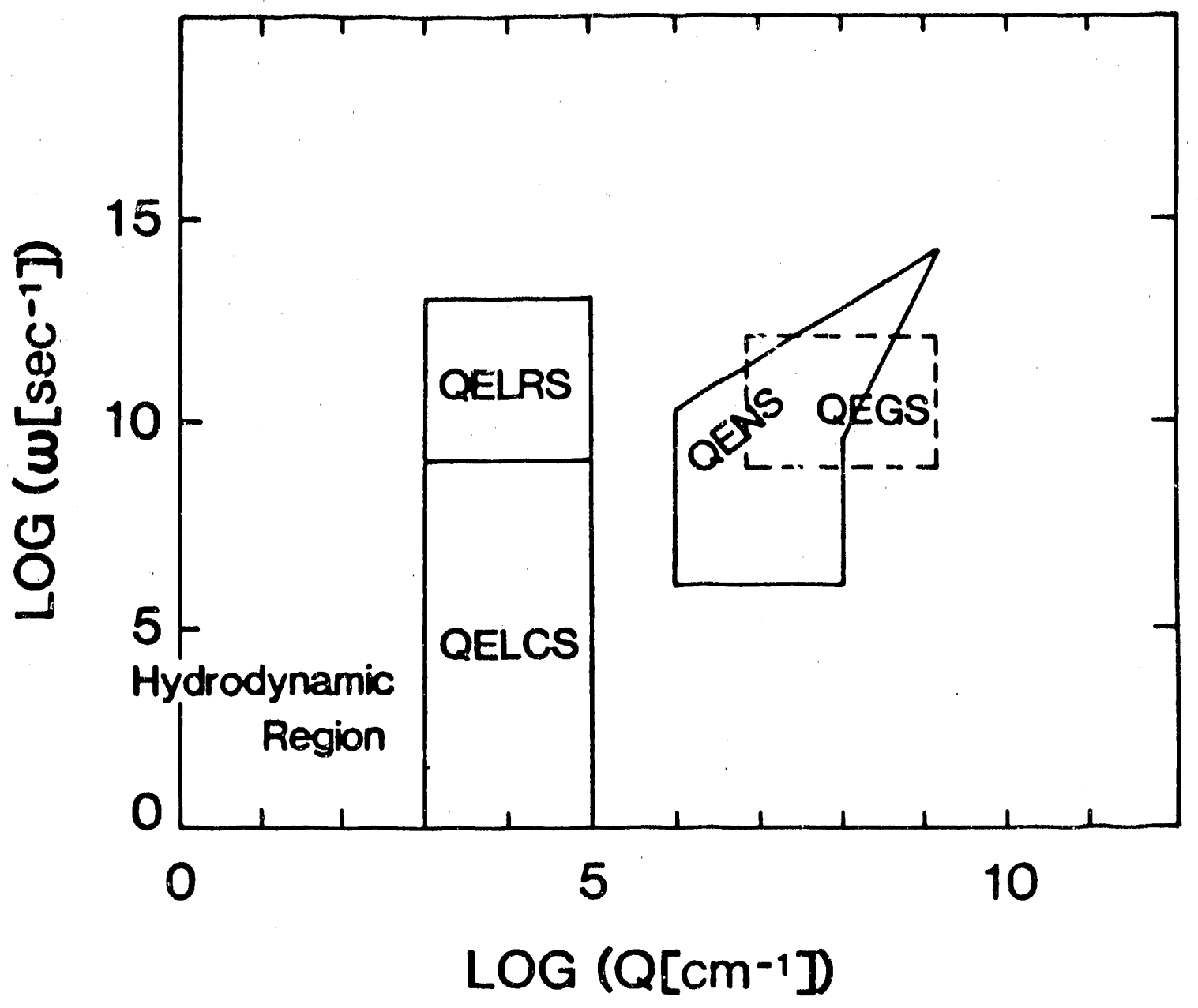

Fig. 1. Diagnostic methods used to investigate the dynamics of liquids: quasielastic light Rayleigh scattering (QELRS), quasielastic light correlation spectroscopy (QELCS), quasielastic neutron scattering (QENS), and quasielastic gamma-ray scattering (QEGS). The computer simulation region (molecular or Brownian dynamics) overlaps with QENS and QEGS. 


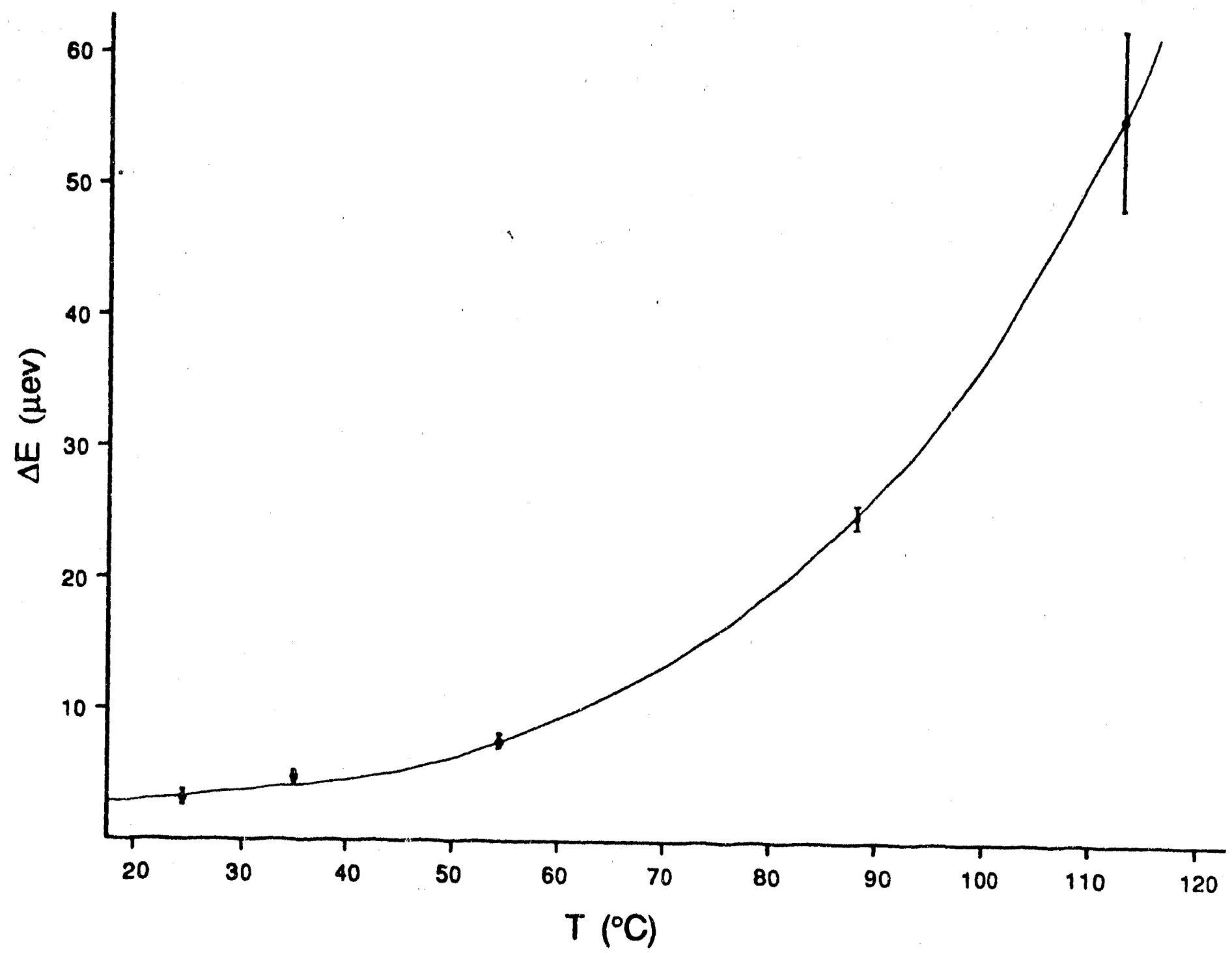

Fig. 2. $\Delta E$, the measured width of the quasielastically broadened line minus the elastic width, in uev versus the temperature in ${ }^{\circ} \mathrm{C}$. 


\section{Renewal Request}

\section{A. Precision measurement of interference in ${ }^{191} \mathrm{Ir}$ and the reexamination of earlier TIV investigations.}

Gimlett et al. ${ }^{1}$ have made a very careful measurement of the phase factor, $\eta$, which would be zero or $\pi$ for time reversal symmetry to hold, for the $129 \mathrm{keV}$ transition in ${ }^{191}$ Ir. Their first results were interpreted with the calculation of interference perfcrmed by Goldwire and Hannon $^{2}$ and appeared to indicate a time reversal invariance violation (TIV) for this case of electromagnetic nuclear decay from a mixed M1-E2 transition.

A mcie detailed calculation of the interference contribution by Davis, Koonin, and Vogl ${ }^{3}$ led to a restoration of time reversal invariarce and appeared to resolve the issue. Our observation of the Mössbauer interference being about $15 \%$ larger in magnitude than that of the Davis et al. calculation raises the question as to the accuracy inherent in these calculations.

We have already attempted to remeasure the Mössbauer lineshape parameters for ${ }^{191} \mathrm{Ir}$ (see Appendix A) and again we have found a magnitude for $\beta$ which appears larger than predicted by the Davis et al. result. Unfortunately, we had to perform this experiment at 77

$\mathrm{K}$ rather than $4 \mathrm{~K}$ and we had to use natural iridium instead of enriched ${ }^{191} \mathrm{Ir}$, which made our errors large enough that a conclusive test of the theory was not possible.

We are now redoing our experiment with some enriched ${ }^{191}$ Ir loaned to us by Dr. F. Wagner, Technische Universität München, in an attempt to reduce our errors. It would be a great asset to this study if D.O.E. would support our request to construct a new source cask capable of attaining $4 \mathrm{~K}$ as we would then be able to obtain a greatly enhanced recoilless fraction and a definitive test of the interference calculations for this important case would then be possible.

- In view of the importance to fundamental physics of finding a case where TIV occurs, besides the famous kaon decay, this experiment will have a high priority in our program. 


\section{References}

1. J.L. Gimlett, H.E. Henrikson, N.K. Cheung, and F. Boehm, Phys. Rev. Lett $\mathbf{4 2}, 354$ (1979).

2. H.C. Goldwire, Jr. and J.P. Hannon, Phys. Rev. B16, 1875 (1977).

3. B.R. Davis, S.E. Koonin, and P. Vogl, Phys. Rev. C22, 1233 (1980).

\section{B. Filtered Source Spectroscopy}

As has been noted earlier ${ }^{1}$ a thick stationary absorber $(t \simeq 10)$ placed between a single line source and a "thin" unsplit moving absorber gives a double pattern ME spectrum (see Figs. 1 and 2 of the Progress Report). Because this technique emphasizes the wings of the spectrum a small interference parameter leads to a noticeable asymmetry in the observed spectrum, which is not visibly apparent in a conventional ME transmission experimest.

We are continuing these experiments to see if this technique can lead to even a more precise determination of the interference parameter $\beta$.

In this work we have found that great care must be taken with enriched powder samples, as they can have significant fluctuations in the thickness of enriched isotope when prepared from powdered samples. This violates the requirement of sample uniformity necessary for a correct fit to the data based on the convolution theorem.

Any noticeable variation in the sample uniformity requirement gives incorrect line-shape parameters. From model calculation we find that these variations in thickness increase the values of $\beta$ and the width $\Gamma$.

We wish to carry out a high precision measurement of the filtered spectrum for the $46.5 \mathrm{keV}$ line of ${ }^{183} \mathrm{~W}$ as a further test of interference parameter calculations and as an independent check of our direct measurement of interference.

An important added check on theory that can be achieved by this technique is the basic correctness of the line-shape-asymtotics. The current wisdom, is that the basic lineshape is Lorentzian with a small asymmetric term that varies inversely with energy in the asymtotic 
region. Previous measurements have been of insufficient accuracy to test this fundamental result. We believe that systematic measurements of the filtered spectra could constitute a confirmation of this widely assumed result.

\section{Study of Phasons in $\mathrm{TaS}_{2}-1 \mathrm{~T}$}

In earlier work ${ }^{1}$ we found evidence of a critical phenomenon in $1 \mathrm{~T} \mathrm{TaS}_{2}$ near the incommensurate to quasicommensurate transition at $79^{\circ} \mathrm{C}$. We have extended these studies using our high intensity ${ }^{183} \mathrm{Ta}$ source and found a sharp increase in the inelastic intensity just about $79^{\circ} \mathrm{C}$. This intensity returns to that below $79^{\circ} \mathrm{C}$ within about $3^{\circ} \mathrm{C}$ about the transition. Atiempts to investigate this anomaly near the Bragg scattering peaks have been difficult because of the small inelastic component, and because of the fluorescence of Ta for the $46 \mathrm{keV}$ line. Also, our original supplier of $\mathrm{TaS}_{2}-1 \mathrm{~T}$ has been unable to provide further samples, although we have recently found a new source of high grade crystals and we intend to pursue these studies further.

The observations to date suggest some type of critical phenomenon, possible a soft mode for phasons associated with the charge density waves in these materials. We hope to utilize a MIcrofoil Conversion Electron (MICE) detector to look for changes in the elastic intensity near the $79^{\circ} \mathrm{C}$ transition, and some new techniques for carrying out these experiments are suggested in the next section.

\section{References}

1. J.R. Stevenson and J.G. Mullen, Sol. State Comm. 39, 319 (1981).

\section{MICE lineshape and a new technique for Doppler shifting the Mössbauer resonance line}

We have carried out MIcrofoil Conversion Election (MICE) experiments with ${ }^{183} \mathrm{~W}$ and demonstrated ${ }^{1}$ a very sustantial enhancement in signal to off-resonance count rates. We have beer able to extend the Fourier-transform method to describe the lineshape and to show that 
to lowest order it is a mirror image of the transmission line-shape. ${ }^{2}$ Because MICE detectors can be made nearly $100 \%$ efficient to resonance radiation while being relatively inefficient to the detection of non-resonance radiation, it is possible to use them in conventional transmission experiments replacing Germanium detectors which are indiscriminate in their efficiency with respect to either resonance or non-resonance radiation. We have beev able to use this technique in ${ }^{183} \mathrm{~W}$ experiments to obtain enhancements of a factor of three in the signal-to-off resonance count rates.

We would like to apply this improveu methodology to problems like $\mathrm{TaS}_{2}-1 \mathrm{~T}$ mentioned earlier, but we have found that the technological difficulties, of moving the cold microfoils at cryogenic temperature, are delicate and require considerable care and servicing of equipment. Thus, since we cannot move our heavily shielded supersources, we are proposing to develop a device for moving the monochromating crystal ( $\mathrm{LiF}$ ) along the bisector of the incident beam and the Bragg scattered beam to effect a Doppler shift in the momentum transfer. It is possible to show that this leads to a Doppler energy shift, $\delta \mathrm{E}$, when the Doppler velocity of the monochromating crystal is $\mathrm{V}$, through the relation.

$$
\mathrm{v}=\left(\frac{\delta \mathrm{E}}{\mathrm{E} \gamma}\right)\left(\frac{1}{2 \sin \theta_{\mathrm{c}}}\right) \mathrm{c},
$$

where $\theta_{\mathrm{c}}$ is the Bragg angle as measured in the crystal reference frame.

We wish to develop this technique so that we can utilize cold MICE detectors without a need for moving the delicate microfoils in the detector and eliminate any complications due to vibration.

With this new technique for Doppler shifting the ME photons, we should be able to carry out a number of here-to-fore difficult and time consuming experiments such as studying the elastic scattering of the elastic component of the $79^{\circ} \mathrm{C}$ transition in $\mathrm{TaS}_{2}-1 \mathrm{~T}$ mentioned in the previous section.

Also, with this improved methodology we would like to make a careful measure of the MICE lineshape and confirm our analysis based on exponential modeling ${ }^{2}$ of the escape of 
electrons from the microfoils of the conversion electron detection system.

\section{References}

1. J.G. Mullen, A. Djedid, C. Holmes, G. Schupp, L. Crow, and W.B. Yelon, Nucl. Inst Methods B14, 323 (1986).

2. J.G. Mullen, A. Djedid, B. Bullard, Hyperfine Interactions 40, 123 (1988).

\section{E. Hyperfine Interaction Evaluated with Fits based on the Convolution theorem:}

\section{application to hydrogenated tantallum.}

The technique of direct fitting of data to the convolution integral can be applied to a wide variety of physical problems. We seek to use this approach to reevaluate our hyperfine spectra for well characterized samples of magnetite ${ }^{1}$ and for complex volcanic basalts ${ }^{2}$ taken from Jeju Island off the coast of mainland Korea.

In addition we are proposing to carry out collaborative research with Professor Dr. F. Wagner of the Techniche Universität München, FRG, to study the recoilless fraction of hyperfine splittings of ${ }^{183} \mathrm{~W}$ in hydrogenated tantalum metal, with the samples being furnished by the Munich group.

Since the presence of hydrogen in this bcc metal will alter the cubic point symmetry a slight quarupole broadening would be expected. This effect can be explicitly incorporated into the convolution integral, and we will also attempt a deconvolution of these spectra using fast Fourier-Transform techniques, augmented by analytic expression for the source spectra distribution. ${ }^{3}$

The widespread potential for using bcc metals as fuel storage cells for hydrogen makes this application particularly interesting. We believe that there are anomalies in the temperature dependence of the recoilless fraction. Our new computational techniques, coupled with the superintense ${ }^{183} \mathrm{~T}$ a sources $(\sim 100 \mathrm{Ci})$ that we have been able to fabricate, places our research 
capabilities in a position that we are uniquely qualified to investigate this system, which has possible practical application with respect to energy storage.

\section{References}

1. G. Haley, J.G. Mullen, J.M. Honig, Sol. State Comm. 69, 285 (1989).

2. S.R. Hong, G. Haley, and J.G. Mullen, Hyperfine Interaction 57, 2221 (1990).

3. J.G. Mullen, A. Djedid, D. Cowan, G. Schupp, M.L. Crow, Y. Cao, and W.B. Yelon, Physics Lett, A127, 242 (1988).

\section{F. Proposed Scattering Experiments}

The work of the past year has demonstrated that the QEGS technique has broad applicability to diffusive systems and solid state transitions. We propose to build upon this with further work in polymeric systems as well as with glasses in which the diffusing species is a heavy ion and consequently has a large scattering cross section. This latter effort was previously proposed, but due to limited time was not performed in this proposal period. In addition, we propose to continue the work on $\mathrm{NiAl}$ and attempt to improve upon the earlier result.

In the process of carrying out these studies, we have become aware of several needs associated with the present system. A recent article by Burrell ${ }^{1}$ pointed out that the uncertainties calculated in many non-linear least squares fitting routines use a "linear" formula and can be significantly smaller than those correctly calculated. This is particularly true when the functional form of the curves being fitted are rather complicated. In work done this summer (1990) by a University of Missouri-Columbia undergraduate, it was found that the uncertainties from our Lorentzian fitting routine were smaller by roughly a factor of 2 than the true uncertainties. Thus we find that higher statistical accuracy is required of the data to achieve the desired certainity: of fit, exacerbating the already difficult problem of adequate 
research time with the QEGS facility. A variety of improvements in the instrument will be needed to overcome these limitations.

First, we propose to rebuild the source cask with multiple beam exit ports and with an improved source cooling system to reach $\sim 10 \mathrm{~K}$ rather than the present $77 \mathrm{~K}$. The multiple ports will allow us to conduct more than one experiment simultaneously. We intend to equip one port with the existing experimental set-up while a second will be set up with a dedicated liquids- amorphous beam, which requires lower $Q$ space resolution and can thus gain in intensity.

Cooling the source to $10 \mathrm{~K}$ (probebly with a closed-cycle refrigerator) will lead to a small improvement in the recoilless fraction in ${ }^{183} \mathrm{~W}(46.5 \mathrm{keV})$, but will have very major significance for the use of higher energy Mössbauer transitions and especially the $103 \mathrm{keV}$ transition in ${ }^{153} \mathrm{Eu}$. This transition is particularly interesting for several reasons. First, this gamma-ray, produced in the $46.8 \mathrm{hr}$ decay of ${ }^{153} \mathrm{Sm}$ is $\sim 20$ times narrower in energy than the ${ }^{183} \mathrm{~W}$ transition and will be especially useful for the solic state transition studies where greater energy resolution is required. Secondly, the decay of ${ }^{153} \mathrm{Sm}$ is especially clean and any instrument using this source will be able to use the direct beam rather than requiring a monochromating filter with its significant intensity loss. Next, the ${ }^{153} \mathrm{Sm}$ sources can be prepared with $\sim 30$ times the activity of the Ta sources, and the decay of ${ }^{153} \mathrm{Sm}$ gives $\sim 5$ times the gamma yield (per decay) compared to ${ }^{183} \mathrm{Ta}(28 \%$ vs. $6 \%$ ) because of the simpler decay scheme and reduced internal conversion. Finally, lower photoelectric absorption in source, windows, etc., gives a greater unable beam. All of these factors lead to experimentally useful fluxes approximately 3000 times more intense than with ${ }^{183} \mathrm{~W}$. If a recoll-free fraction of $3 \%$ can be achieved, then the effective beam will be $\sim 300$ times greater than with $W$. However, at $77 \mathrm{~K}$, the recoilless fraction of ${ }^{153} \mathrm{Sm}_{2} \mathrm{O}_{3}$ is only a fraction of a percent. Therefore cooling to $10 \mathrm{~K}$ is crucial.

The source cask will actually be designed with $4-90^{\circ}$ ports, as this extra access adds minimal cost to the project. Space limitations of the present site of the QEGS facility would 
allow the use of at most three of these and we inagine that the third port will be used primarily for the development of techniques such as new detectors, and piezoelectrically modulated monochromators which would allow velocity (energy) scanning with a stationary detector. This would help our effort with the conversion electron detector for which reliability is presently low due to the need to oscillate the cooled assembly. ${ }^{2}$ The competing demands of technique development and liquids and solids research would thus be met and enable us to make for greater progress.

\section{References}

1. K. H. Purrell, Amer. Journ. of Phys. 58 160, (1990),

2. J.G. Mullen, A. Djedid, C. Holmes, G. Schupp, L. Crow and W. Yelon, Nucl. Inst. and Methods B14, 323 (1986).

\section{G. Proposed Colaboration on Polymers and Glassy Systems.}

The major part of our research in polymers and glassy systems will be in collaboration with Dale Schaefer from Sandia National Laboratory. That work is part of a larger program of elastic and inelastic scattering to be conducted partially at Argonne National Laboratory and other national laboratories. This is described below and we believe that it will form the basis for a substantial continuing collaboration.

The nature of glass materials has intrigued and mystified physicists for decades. In spite of substantial research effort, a microscopic understanding of the origin of glassy behavior has proved elusive. Unexplained similarities exist between separate classes of glasses surh as atomic, molecular, polymeric and network glasses. In spite of this seeming universality, distinct differences exist between so called strong and fragile glass-formers. Moreover, the unusual low-temperature thermal properties of glasses signify low-energy vibrational excitations that are largely unexplored and poorly understood. Finally, the nature of the glass 
transition itself remains controversial with competing models based respectively on purely kinetic entrapment ideas and critical-like divergences as predicted by mode-coupling theory.

It is our opinion that the nature of both the glassy state and the glass transition can be substantially illuminated by elastic and inelastic neutron scattering covering a large range of $\mathrm{Q}-\omega$ space and by QEGS. In particular, we find it conceptually convenient to separate. the structural and topological properties of glasses. Whereas powder diffraction is sensitive exclusively to structure (ie the position of the atoms), inelastic scattering is sensitive to the force field in which the atoms lie. The latter, therefore, at least in principle, provides a handle on the elusive topological nature of glasses. We have already demonstrated that exceedingly low-energy excitations are found in porous silica aerogels and have provided reasonable evidence that these excitations are related to network topology using the concept of fractons (the analogue of phonons on a network).

We propose to investigate the structure and dynamics of several classes of materials using a variety of instruments and several international facilities. This project will require a variety of neutron scattering techniques, including powder diffraction over a wide range in Q, small angle scattering and inelastic scattering at very high energy resolution as well as the quasielastic $\gamma$-ray techniques. (D.W. Schaefer and B.J. Olivier).

Dr. Schaefer is preparing a large number of samples for characterization with neutrons and the most appropriate ones will be sent to Missouri for QEGS studies. They will be selected from the following categories:

1. Silica Gels of Varying Connectivity

2. Percolating Network Glasses

3. Tenuous Solids from Linear Polymers

4. Glassy Behavior in Disrupted Network

5. Dynamics of Cross-linked Rubber

6. Se-Ge and Related Glasses 


\section{Budget}

Although the diffraction Mössbauer experiments to date have been very successful and hold forth the promise of still more exciting results, the most limiting factor in progress now is manpower. At present only one graduate student, Ralph Wagoner, is supported on the grant (45199). There are two additional Purdue students who are working on the project, but without support, and it may be necessary to put them on another project if funding to support them is not forthcoming.

At UMC graduate students have been sought, since Lowell Crow completed his thesis in 1987, but none of these have worked out and there are presently no graduate students on the project from the Columbia campus. This means that if we do not get support for the three Purdue students there may be only one student on the project soon, and this will have a crippling effect on what can be done. Most of the funding sought by the principal investigator (JGM) is for the support of these three students and travel money to allow them to spend long stays in Columbia using the MURR facility.

It should be noted that the cost of the equipment requested is being shared by Purdue University, so that for every dollar that is cut out of the budget for equipment two dollars will be lost to the Purdue research equipment budget. Purdue's support for this project is shown by the willingness to cost share equipment, and Purdue has subsidized this project in the present and past. The Physics department bought two computer stations for the project, has paid four months of Bruce Bullard's salary when he was finishing his thesis, and has covered fiscal year deficits since expenses have always exceeded funds granted to Purdue.

The need to augment our technique with He cryogenics is the next biggest item in priority besides support of graduate students. Several experiments that are not possible for higher energy $\mathrm{ME}$ transitions like ${ }^{153} \mathrm{Sm}$ become feasible at $4-10 \mathrm{~K}$.

We have budgeted the cost of renting an apartment, where my graduate students and I can stay during our visits to Columbia. At this time I estimate that each of my students as 
well as myself will average at least two months per year at Columbia, and an apartment is cheaper and superior to motels for this purpose.

We believe that our track record over the last three years is strong, and, when compared with other DOE sponsored research, warrants the appropriation of the requested funding. The large number of publications, invited talks, and $\mathrm{Ph} . \mathrm{D}$ 's coming out of the project all indicate a vigorous program worthy of the support level sought. 


\section{FROFOSED BUDGET \\ U. S. DEPARTMENT OF ENEFGY \\ $03 / 01 / 91-02 / 28 / 94$}

YEAR 1 YEAF 2 YEAR 3

TOTAL

I. SALARIES \& WAGES

A. Principal Investigator

J. G. MULLEN 16.7\%FY

12964

14001

15122

B. Grad Assistant in Fesearch

2 a $50 \% \mathrm{FY} 12 \mathrm{mo}$.

1 a 50\%FY $9 \mathrm{mo}$.

(yr. 1,9 ma: yr 2 \& 3,12 mo

25048

9494

TOTAL SALAFY \& WAGES

II. GRAD FEE REMITS

2 a $\$ 135 / M 0 . \times 12$

1 D $\$ 135 / \mathrm{MO} \times 9$ (YFi. 1 )

1 a $\$ 135 / M 0 \times 12$ (YR.'2 \& 3)

TOTAL FEES

II I. FRINGE BENEFITS

$\begin{array}{rr}\text { A. } & 27.1 \% \\ \text { B. } & 1.8 \%\end{array}$

TOTAL FRINGE BENEFITS

IV. TRAVEL: Domestic

a Trips to Univ. of Missouri

Lodging at $U$. Missouri

50 Per diem a\$24/day

Car rentals

3240

1215

4455

3513

621

$41 \geq 4$

3240

1620

3240

1620

4860

4860

3794

711

4505

4099

746

4845

1 Trip AFS Meeting

1 Airfarelyr.

5 Lodging J \$75/day

5 Fer diem $2 \$ 24 /$ day
4850

1200

530

4625

1200

280

400

375

120

425

375

120
5000

1200

605

450

375

120

750

540

192

268

250
0

0

7500
7000

0

0

0

TOTAL DOMESTIC TRAVEL
1 Airfare
6 Lodging a $\$ 90$
8 Per diem a $\$ 24 /$ day
Car rental
Registration
14175

42087

78964

36452

157503

9720

4455

11406

2078

$1 \$ 484$

14475

3600

1415

1275

1125

360

750

540

192

268

250

24250 
Foreign Travel

1 Trip

Nanjing CHINA $(9 / 2-9 / 6 / 91)$

6 Days Do $150 /$ day

1500

0

900

Registration

250

Ground Transp.

350

TOTAL FOREIGN TRAVEL

3000

0

0

1500

900

250

550

5000

V. PUELICATIONS

10 PAGES D \$5O/FAGE/YR.

500

500

500

1500

VI. QTHEF SUFPLIES \& EXPENSE (Hi storical)

A. Communx cations (1 ong

$$
\text { distance (alls) }
$$

B. Xeroxing, duplicating, drafting, electronics. shopwork, etc. TOTAL OTHEF DIFECT COST

\section{0}

500

1000

2500
1000

500

1250

2750
1000

500

1500

3000
3000

1500

5750

8250

VII. EQUIFMENT.

Liquid Helium Cryagenics 9300

Germaman deetector / fast electronics

2 Liquid He. Dewar

2 Liquid Nitrogen Dewars

Vacuum Facility

3500

1500

9300

3500

1500

1000

1000

2500

2500

TOTAL EQUIFMENT

9300

5000

5500

17800

VIII. TOTAL DIFECT COST

VIII. INDIFECT COST 49\% MTDC

IX. TOTAL COST

\begin{tabular}{|c|c|c|c|}
\hline 78.595 & 78566 & 83001 & 239962 \\
\hline$=========$ & $\begin{array}{c}33666 \\
======\end{array}$ & $\begin{array}{c}36574 \\
=====\end{array}$ & $\begin{array}{r}101914 \\
======\end{array}$ \\
\hline 110069 & 112232 & 119575 & 341876 \\
\hline
\end{tabular}


Please Print of Type

\section{Organization}

Purdue Research Foundation

Deincidar invesiloalo IP / /Prolec: Direcior IP D I

James G. Mullen

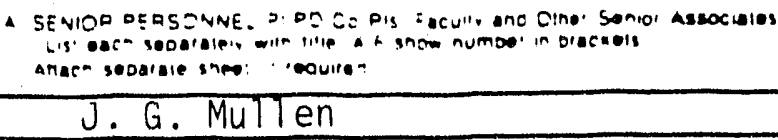

1 TC:a. jeniog ofasonne.

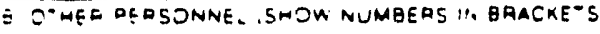

DOS: DOR: DAA. ASSOCIATES

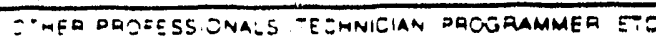

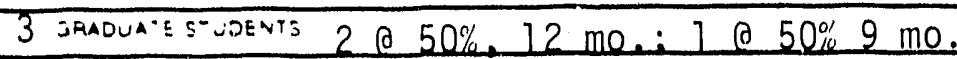

一 JNDEOERASUA-E : TUDEN"S

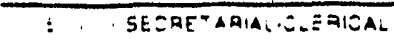

$T$ TIAEO

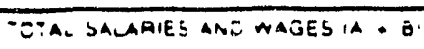

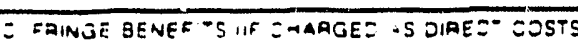

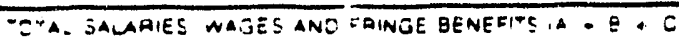

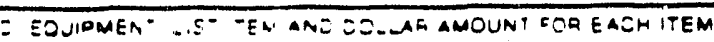
Liquid Helium Cryogenics
$\$ 18,600 *$

-otal equipmen *Department mat.ching funds $\$ 9,300$

E taavel . domestil IInC. ganada ano us possessions!

: Foretor Nanjing CHINA, Seft.. $2-6,1991$

\footnotetext{
E DTHER DIAE:: :OSTS

- matepials ano supo! ies

: Publication gosistrage EmaRge:

J CONSULTANT SERVICEs

- COMPUTER IAOPE. SERVICEs

S SONTAAC-S ANO SUBGRAMTS

s OTME Grad Fee Remits

TOTAL JPAEA OIAEC- SOSTE

I TOTAL JIAEC COS: ${ }^{-}$A HROUGM

- INOIAECi COSTS ISPEEIEV QATE AND BASE,
}

Total INDIAEE sos? $\quad 49 \%$ of all except D \& F6

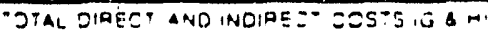

- applienanis zzsi smapinu if ant

a TOtal amoun: ó -ais aEOUES: IITEN, LESS ITEM.

PI: DD TYPED NAME \& SIGNATURE

James G. Mullen \& amar \&. Mule

INST RED TYPED NAME \& SIGNATURE
FOR DOE USE EN.

Prodosal No

Awaro No

$02 / 28 / 92$

\begin{tabular}{l}
$\mid \begin{array}{l}\text { DOE Funded } \\
\text { Person.Mos }\end{array}$ \\
\hline S.
\end{tabular}

Eunas Requesioc

IV ADDlicant

\begin{tabular}{l|l|l}
\hline 2.0 & 1 & $T 2,964$ \\
\hline
\end{tabular}

176.5

47,506

4,134

51,640

34,542

18,600

7,000

3,000

2,500

500

4,455

7,455

37,695

31,674

119,369

y. 300

110,069

DATE

$10 / 04 / 90$

DATE 


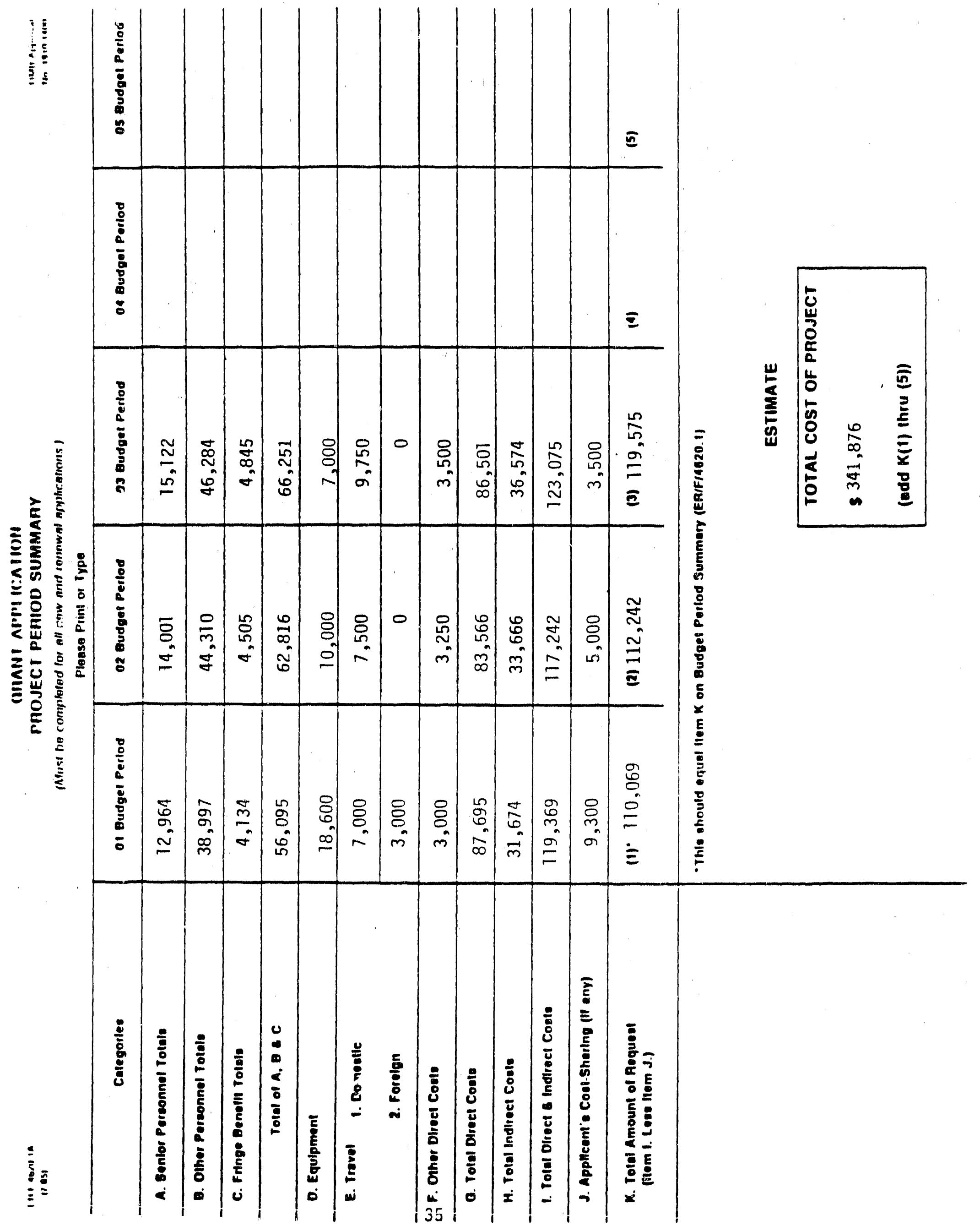




\begin{abstract}
We give a progress report for the work which has been carried out in the last three years with D.O.E. support. A facility for high-intensity Mössbauer scattering is now full operational at the University of Missouri Research Reactor (MURR) as well as a facility at Purdue, using special isotopes produced at MURR. High precision, fundamental Mössbauer effect studies have been carried out using scattering to filter the unwanted radiation. These have led to a new Fourier transform method for disscribing Mössbauer effect (ME) lineshape and a direct method of fitting $\mathrm{ME}$ data to the convolution integral. These methods allow complete correction for source resonance self absorption (SRSA) and the accurate representation of interference effects that add an asymmetric component to the $\mathrm{ME}$ lines. We have begun applying these techniques to attenuated ME sources whose central peak has been attenuated by stationary resonant absorbers, to more precisely determine interference parameters and line-shape behavior in the resonance asymptotic region. This analysis is importani to both the fundamental ME studies and to scattering studies for which a deconvolution is essential for extracting the correct recoilless fractions and interference parameters. A number of scattering studies have been successfully carried out including a study of the thermal diffuse scattering in $\mathrm{Si}$, which led to an analysis of the resolution function for gamma-ray scattering. Also studied was the anharmonic motion in $\mathrm{Na}$ and the satellite reflection Debye-Waller factor in $\mathrm{TaS}_{2}$, which indicate phason rather than phonon behavior. We have begun quasielastic diffusion studies in viscous liquids and current results are summarized. These advances, coupled to our improvements in MIcrofoil Conversion Electron spectroscopy lay the foundation for the proposed research outlined in this request for a three-year renewal of D.O.E. support.
\end{abstract}

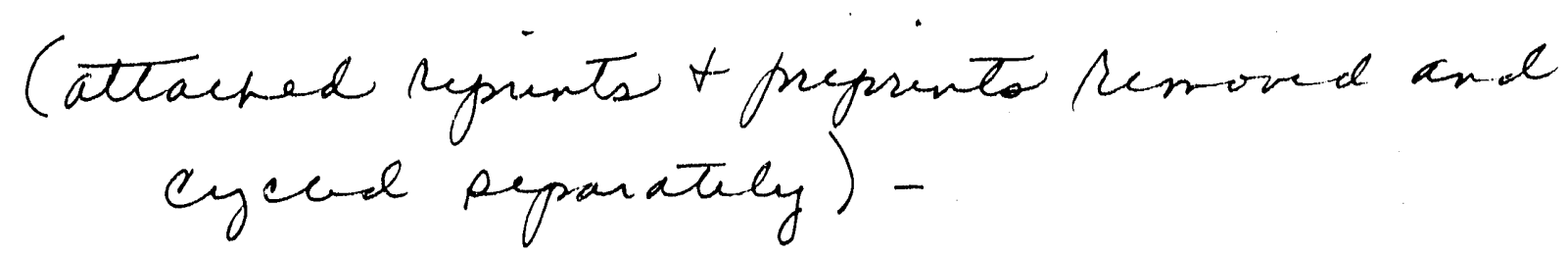




\section{DISCLAIMEK}

This report was prepared as an account of work sponsored by an agency of the United States Government. Neither the United States Government nor any agency thereof, nor any of their employees, makes any warranty, express or implied, or assumes any legal liability or responsibility for the accuracy, completeness, or usefulness of any information, apparatus, product, or process disclosed, or represents that its use would not infringe privately owned rights. Reference herein to any specific commercial product, process, or service by trade name, trademark, manufacturer, or otherwise does not necessarily constitute or imply its endorsement, recom. mendation, or favoring by the United States Government or any agency thereof. The views and opinions of authors expressed herein do not necessarily state or reflect thos of the United States Government or any agency thereof. 

